\title{
Inflammatory bowel disease and the lung in paediatric patients
}

The prevalence of inflammatory bowel disease (IBD) has increased over the past 20 years. Pulmonary involvement in paediatric IBD is rare but may be missed since the spectrum of symptoms is broad and mimics other diseases. The most important differential diagnoses of pulmonary manifestations of IBD are infections and therapy-related side-effects. There is no gold standard to diagnose respiratory manifestations in children with IBD. Diagnostic tests should be chosen according to history and clinical presentation. Treatment of respiratory manifestations of IBD includes inhaled or oral corticosteroids and initiation or step-up of immunomodulatory IBD therapies.
An estimated 7 million people worldwide suffer from inflammatory bowel diseases (IBD) [1]. Over the past two decades, the age-standardised prevalence of IBD has significantly increased to more than 200 cases per 100000 population in high-income societies [1]; this increase affected both adults and children [2]. $20 \%$ of patients affected by IBD are children [3]. 7-10 out of 100000 children in Western societies develop IBD in any given year $[4,5]$. IBD is an umbrella term and includes Crohn's disease (CD) and ulcerative colitis (UC). Both types affect children usually older than 6 years of age [3]. Special attention should be paid to linear growth impairment, malnutrition, delayed puberty, bone mass density and mental health in children with IBD [6].

$21-41 \%$ of patients with IBD present with an extraintestinal manifestation at least once in their lifetime [7]. Knowledge about pulmonary involvement in paediatric IBD is scarce and its exact prevalence is unknown [8]. In adults, the prevalence of respiratory involvement in IBD patients is estimated at $40 \%$. The majority of findings are nearly or completely asymptomatic and of uncertain significance for the long-term course [9]. The spectrum of symptoms is broad and may mimic other diseases. The differentiation between primary pulmonary manifestations and pulmonary side-effects attributable to the therapy for IBD can be challenging [10].

\section{Respiratory manifestations of IBD}

Every part of the respiratory system including upper and lower airways, lung parenchyma, and the pleura can be affected by IBD [11]. In adults, respiratory manifestations most commonly present with bronchiectasis and chronic bronchitis in 20\% of IBD patients. Interstitial lung disease, organising

@ERSpublications

Pulmonary involvement in paediatric IBD is rare but may be underdiagnosed. The spectrum of symptoms is broad and mimics other diseases. The differentiation between IBD-related and druginduced pulmonary manifestation can be challenging. https://bit.ly/3uZBvpA

Cite as: Jochmann A, Trachsel D, Hammer J. Inflammatory bowel disease and the lung in paediatric patients. Breathe 2021; 17 : 200269. 
pneumonia and peripheral nodules are less common respiratory manifestations of IBD $[11,12]$.

Interstitial lung disease seems to be the most common respiratory manifestation of IBD in children according to the published literature [13] In our own limited experience, IBD seems to affect the upper and lower airways at least as often than the lung parenchyma. Current knowledge of respiratory manifestations of IBD in children originates from single case reports and case series. Large epidemiological or clinical studies are missing. The prevalence of respiratory manifestations is in the range of $3-10 \%$ according to data from computed tomography $(\mathrm{CT})$ or questionnaires in children with IBD [14, 15]. Respiratory symptoms and lung disease may precede the classical gastrointestinal presentation of IBD in both children [13] and adults [11, 16]. Early recognition and correct diagnosis of respiratory manifestations of IBD are important because they are often strikingly responsive to corticosteroid treatment [17].

\section{Airway involvement}

Upper airway involvement may present as atypical croup with cough, hoarseness, stridor and even severe upper airway obstruction. Endoscopy may show erythema and small, patch-like (sub)-mucosal granulations in the large airways. Biopsy may confirm granulomatous disease. Chest CT can detect nodular thickening of the tracheobronchial wall. Upper airway obstruction can be so severe and life-threatening that it may require intubation. It usually responds well to treatment with corticosteroids [18-20].

Symptomatic lower airway involvement is much more common in adults than in children. Bronchiectasis can be present in up to $75 \%$ of adult IBD patients [17]. In contrast, bronchiectasis has been very rarely described in paediatric patients. It may manifest as purulent bronchitis and seems to respond well to corticosteroid treatment [21].

\section{Pulmonary parenchymal disease}

In one large paediatric case series including 16 patients, interstitial lung disease was the dominating respiratory manifestation of CD [13] Diffuse bilateral interstitial disease was noted in $69 \%$ of patients. A rare interstitial manifestation was found in a 17-year-old female CD patient who presented with bilateral cavitary pneumonia. She was unresponsive to antibiotics but improved with infliximab therapy [13]. Seven of the 16 patients had cryptogenic organising pneumonia [22-27]. Two of these had interstitial pulmonary fibrosis [22, 24]. Five out of 16 patients presented with single lobe consolidations among whom one patient, an 11-year-old girl, had no prior history of IBD [13]. Bilateral pneumonia and peripheral non-caseating epithelioid granulomas mimicking sarcoidosis may also occur in paediatric IBD [28, 29].

\section{Pathophysiology}

The pathogenesis of pulmonary involvement in IBD is not well understood. Molecular mimicry or aberrant cell homing from the shared embryological origin of the pulmonary and intestinal system from the primitive foregut may play a role in the disease process [30]. Colonic and respiratory epithelia express submucosal lymphoid tissue and are important for host mucosal defence mechanisms. Hypothetically, inflammatory processes in intestinal and respiratory tissue could result from epithelial exposure to common antigens by inhalation or ingestion, and lead to sensitisation of lymphoid cells and to a similar response in lung and gut tissue [31]. Another hypothesis is that antigen leakage through the impaired intestinal barrier increases local and systemic inflammatory responses [32]. In an animal model of UC, the lungs and the colon both showed increased concentrations of proinflammatory cytokines like vascular endothelial growth factor (VEGF) and tumour necrosis factor (TNF)- $\alpha$. The cytokine-mediated increase in alveolar epithelial permeability may play a role in the pathogenesis of the pulmonary involvement in IBD [32]

Human leukocyte antigens (HLAs) have been linked to extraintestinal manifestations of IBD in the skin, joints and eyes. This has been described for HLA-A2 and HLA-DR1 in CD; and HLA-B27, HLAB58 and HLA-B8/DR3 in UC [33, 34]. No genetic predisposition for respiratory manifestations of IBD has yet been found [35].

\section{Histopathology}

Lymphocytic, mononuclear or polymorphonuclear cell infiltration and mucosal ulcerations are characteristic for IBD-related inflammation of the respiratory system. Non-caseating granuloma formation may occur in the airway mucosa and the lung parenchyma [13, 14, 18, 25, 29]. IBDrelated interstitial lung disease usually presents as cryptogenic organising pneumonia. Rarer forms include neutrophilic necrotic parenchymal nodules without signs of infection and serositis [36].

Acute alveolitis with severe dysplasia of pneumocytes and interstitial fibrosis has been described in biopsies of a paediatric and an adult patient with $C D$. Both suffered from CD for more than 6 years and presented with shortness of breath 2 years after resection of the terminal ileum. Immunohistology identified predominantly lambda chains expressed by lymphocytes associated with $\lg A$ and IgM [25].

Infiltration of lung tissue with eosinophils and eosinophilia in peripheral blood should prompt consideration of a drug-induced pulmonary pathology [37]. 


\section{Pulmonary side-effects of IBD therapies}

It may be difficult to differentiate respiratory manifestations of IBD from respiratory symptoms related to the treatment of IBD. Various treatment options exist for paediatric IBD including sulfasalazine, 5-aminosalicylic acid (5-ASA), parenteral nutrition, oral corticosteroids, azathioprine, methotrexate, antibiotics, surgery, and most recently, antiTNF- $\alpha$ monoclonal antibody therapy [38]. Immunosuppressive therapy with sulfasalazine can result in eosinophilic pneumonia and rarely fibrosing alveolitis [37]. Fibrosing alveolitis is a very rare, potentially lethal side-effect that has been described in adult IBD patients. Eosinophilic pneumonia typically occurs 2-6 months after initiation of therapy with sulfasalazine [37]. Pneumonitis with dyspnoea, cough, fever, hypoxaemia and interstitial infiltrates has been described in adult patients under methotrexate (the majority of whom were affected with rheumatoid arthritis) [39].

Biologicals are increasingly used in paediatric IBD. Anti-TNF- $\alpha$ antibodies, such as infliximab and adalimumab, have been described to cause acute infusion reactions, delayed hypersensitivity reactions and opportunistic infections, and to pose a potential risk for malignancies [38]. Mild infections, especially of the gastrointestinal and respiratory tract, have been reported in paediatric $C D$ patients treated with infliximab or adalimumab [40]. There is a risk of reactivation of tuberculosis and other mycobacterial infections under anti-TNF- $\alpha$ therapy, especially in adults [41]. The majority develop extrapulmonary tuberculosis. All patients should be actively screened for latent tuberculosis before initiation of anti-TNF- $\alpha$ treatment.

In adults, Pneumocystis jirovecii pneumonia has been described under treatment with infliximab [42]. To our knowledge, there are no clear recommendations for considering $P$. jirovecii pneumonia prophylaxis in children with IBD under immunosuppressive therapy.

Fungal infections have also been related to antiTNF- $\alpha$ therapy in adult patients [43]. Opportunistic infections due to immunosuppressive treatment with anti-TNF- $\alpha$ therapy, azathioprine or methotrexate must be ruled out before treating pulmonary abnormalities as extraintestinal manifestations of IBD. Patients with monotherapy of azathioprine, corticosteroids or anti-TNF- $\alpha$ therapy have a threefold higher risk for an opportunistic infection compared with patients without immunosuppressive therapy [44]. Combination therapies potentiate this risk. Apart from Mycobacterium tuberculosis, causative agents include P. jirovecii, Listeria monocytogenes, Aspergillus fumigatus, Cryptococcus neoformans or cytomegalovirus [37].

Rare non-infectious pulmonary side-effects of immunosuppressive therapy like alveolitis, nonspecific interstitial pneumonitis or diffuse alveolar haemorrhage have been described in adult patients only. These mostly resolve after discontinuation of therapy [45].

A controversial aspect of IBD therapy is the occasional occurrence of pulmonary involvement after colectomy. The debate is whether colectomy itself increases the risk of pulmonary involvement, for example, because the inflammatory process may shift from the gastrointestinal tract to the airway [46]. Another hypothesis is that late pulmonary involvement reflects the reduction of immunosuppression after surgery allowing pulmonary disease to flare up [36, 47]. It could also be speculated that disease activity is not necessarily synchronous among the involved organs, as reflect by abnormal pulmonary findings among IBD patients in clinical intestinal remission [48].

\section{Diagnosis of respiratory involvement (except lung function testing)}

The ESPGHAN (European Society for Paediatric Gastroenterology, Hepatology and Nutrition) task force does not recommend regular screening for respiratory disease in children with IBD [49, 50].

A thorough medical history including a precise description of symptoms, the course of the symptoms, accompanying and environmental factors, and the current and past medication is key in children with respiratory complaints. A gastrointestinal IBD exacerbation can also be the cause for respiratory symptoms. In case of suspicion, laboratory studies may help to confirm the presence of an IBD exacerbation [45].

Other respiratory diseases like tuberculosis or granulomatosis with polyangiitis may mimic respiratory manifestations of IBD in children. This may require further disease-specific laboratory investigations such as interleukin (IL)-6, serological tests, rheumatoid factor, antinuclear antibodies, antineutrophil cytoplasmic antibody and interferon- $\gamma$ release assay (IGRA) [51].

Children with severe respiratory involvement like bronchiectasis should undergo a thorough immunological workup to exclude underlying immunodeficiency or rare syndromes, such as the IPEX syndrome (immunodysregulation, polyendocrinopathy, enteropathy X-linked syndrome).

Side-effects of medications should always be considered as a cause of respiratory disease in patients with IBD, especially in patients receiving biologicals. Sputum culture and nasopharyngeal swabs can help to identify infectious causes of respiratory disease in children with IBD. It very important to rule out atypical infections, such as mycobacteria and fungi, as a complication of immunosuppressive treatment.

Imaging, especially chest $\mathrm{CT}$, plays an important role in diagnosing pulmonary involvement in 
adult IBD [9, 52]. The most common findings are bronchiectasis, bronchial wall thickening, lung opacities, emphysema and ground-glass opacities. Lung parenchymal involvement is reported more frequently for UC than for CD with a female preponderance. It often manifests with consolidations referred to as organising pneumonia [53]. Another rare parenchymal manifestation in adult IBD is cellular nonspecific interstitial pneumonia, which appears as ground-glass opacities with reticulation and a sub-pleural and basal distribution on chest CT. Honeycombing is typically absent [53].

Imaging data for children is only available from case reports. Chest radiography is often normal or reveals unspecific signs of bronchiectatic disease or lung parenchymal disease [17]. Chest CT is not routinely recommended in children due to the rare pulmonary involvement and the exposure to radiation [14, 53]. It classically demonstrates diffuse or localised concentric bronchial wall thickening (with or without mucoid impaction) and bronchiectasis (cylindric, varicose or cystic) [53]. However, imaging may also display the manifold manifestations of IBD lung disease such as basal infiltrates, patchy dense lesions, reticulonodular lesions and lobar pneumonia [13].
Bronchoscopy can be a valuable diagnostic tool to evaluate the large airways, especially in children presenting with croup. Bronchoscopy with bronchoalveolar lavage can provide microbiological specimens to rule out an infectious cause in patients with unspecific respiratory symptoms such as productive cough. Furthermore, mucosal biopsies taken during bronchoscopy can characterise the type of bronchial inflammation [51]. Bronchial, trans-bronchial or open lung biopsies may help differentiating pulmonary manifestations of IBD from autoimmune diseases secondary to treatments with biologicals [45]. The differentiation between sarcoidosis and IBD can be challenging because both can present with non-caseating granulomas [28].

\section{Lung function abnormalities}

In adult IBD, lung function abnormalities found in patients with airway involvement are typically characterised by airflow obstruction without a significant reversibility to bronchodilators. Most patients with parenchymal lung diseases show restrictive lung disease and a reduced diffusing capacity.

Table 1 Studies assessing lung function in children with IBD

\begin{tabular}{|c|c|c|c|}
\hline Lung function technique & Reference & Study population & Finding \\
\hline \multirow{3}{*}{$\begin{array}{l}\text { Body plethysmography } \\
\text { Spirometry }\end{array}$} & {$[57]$} & $12 \mathrm{CD}$ and $18 \mathrm{UC}$ versus 32 controls & No difference \\
\hline & {$[48]$} & $30 \mathrm{CD}$ and 18 UC versus 108 controls & $\mathrm{MEF}_{50}, \mathrm{MEF}_{25}$ reduced in CD \\
\hline & {$[56]$} & $52 \mathrm{CD}$ and $48 \mathrm{UC}$ versus 50 controls & $\begin{array}{l}\mathrm{FEV}_{1}, \mathrm{FVC} \text { reduced } \\
15 \text { restrictive } \\
19 \text { obstructive }\end{array}$ \\
\hline \multirow[t]{4}{*}{$D_{\text {LCo }}$} & {$[14]$} & $25 \mathrm{CD}$ and 25 UC versus 39 controls & No difference \\
\hline & {$[57]$} & 12 CD and 18 UC versus 32 controls & No difference \\
\hline & {$[48]$} & $30 \mathrm{CD}$ and 18 UC versus 108 controls & $D_{\text {Lco }}$ reduced \\
\hline & {$[56]$} & $52 \mathrm{CD}$ and 48 UC versus 50 controls & $D_{\mathrm{LCO}}$ reduced \\
\hline \multirow{3}{*}{$F_{\text {ENO }}$} & {$[57]$} & $12 \mathrm{CD}$ and $18 \mathrm{UC}$ versus 32 controls & No difference \\
\hline & {$[14]$} & 25 CD and 25 UC versus 39 controls & $F_{\mathrm{ENO}}$ higher in UC \\
\hline & [55] & $30 \mathrm{CD}$ and 30 asthma versus 20 controls & $\begin{array}{l}\text { No correlation between } \\
F_{\text {ENO }} \text { and CD activity }\end{array}$ \\
\hline Lung clearance index & {$[57]$} & $12 \mathrm{CD}$ and $18 \mathrm{UC}$ versus 32 controls & No difference \\
\hline \multirow[t]{2}{*}{ Exhaled breath condensate } & {$[54]$} & $22 \mathrm{CD}$ and 25 UC versus 37 controls & $\begin{array}{l}\text { IL- } 6, \text { TNF- } \alpha, \text { IL-1 } \beta \\
\text { significantly higher in IBD }\end{array}$ \\
\hline & {$[55]$} & 30 CD and 30 asthma versus 20 controls & $\begin{array}{l}\mathrm{pH} \text { lower in IBD and asthma } \\
\text { 8-isoprostane differed } \\
\text { between groups }\end{array}$ \\
\hline
\end{tabular}

$\mathrm{MEF}_{50 / 25}$ : maximum expiratory flow at 50/25\% of FVC; $\mathrm{FEV}_{1}$ : forced expiratory volume in $1 \mathrm{~s}$; FVC: forced vital capacity; BHR: bronchial hyperresponsiveness; $F_{\mathrm{ENO}}$ : fractional exhaled nitric oxide. 
However, the variety of lung functions alterations is as manifold as the clinical manifestations [37].

Most studies on respiratory manifestations of IBD in children include lung function testing. To date, there are six studies that assessed lung function in 294 children with IBD [14, 48, 54-57]. Pulmonary function abnormalities were generally inconsistent and mild. There is no correlation with IBD activity (table 1). The main findings include some degree of bronchial obstruction and/or reduced diffusing capacity of the lung for carbon monoxide $\left(D_{\mathrm{LCO}}\right)$. As a note of caution, anaemia is common in IBD and $D_{\mathrm{LCO}}$ measurements need to be corrected for haemoglobin levels. Asthma is more prevalent in male children with IBD and can be identified by pulmonary function testing [58].

\section{Management}

An escalation of the current therapy of IBD should be considered if respiratory manifestations arise. Treatment plans for primary respiratory manifestations of IBD are mostly derived from case series. Bronchial inflammation with or without bronchiectasis, which is unresponsive to antibiotics, should be treated with inhaled or oral corticosteroids. Organising pneumonia has been successfully treated with oral corticosteroids, although sometimes spontaneous remission can be observed [9]. Infliximab has been used successfully for the treatment of organising pneumonia and bilateral cavitary pneumonia [59,60].

Upper airway involvement with severe tracheal inflammation and obstruction of the tracheal lumen has shown dramatic improvement following treatment with intravenous methylprednisolone $[18,61]$. It can lead to irreversible stenosis if it remains untreated [53].

Interstitial lung disease related to IBD responds well to treatment with oral corticosteroids or infliximab [13, 37]. Alternative IBD treatment regimens need to be discussed if the patients suffer from drug-induced respiratory complications [45].

\section{Self-evaluation questions}

1. Which of the following statements about respiratory involvement in children with IBD is/are true?

a) Every part of the respiratory system (including the upper and lower airways, the lung parenchyma, and the pleura) can be affected.

b) The respiratory tract is affected in at least $50 \%$ of children with IBD.

c) ACT scan is mandatory if respiratory symptoms occur in children with IBD.

d) Respiratory manifestation of IBD can be ruled out by negative inflammatory parameters in blood tests.

2. Which of the following statements about the pathophysiology of respiratory manifestations of IBD is wrong?

a) Epithelial exposure to common antigens by inhalation or ingestion could lead to sensitisation of lymphoid cells and a similar inflammatory response in lung and gut.

b) Increased concentrations of proinflammatory cytokines like VEGF and TNF- $\alpha$ can be found in the gut and the pulmonary tissue.

c) A genetic predisposition for respiratory involvement has been identified in IBD patients.

d) The impaired intestinal barrier could result in more antigens entering the body and causing local and systemic inflammation.

3. Which lung function test can exclude respiratory disease caused by IBD in children?
a) Body plethysmography
b) $D_{\mathrm{LCO}}$
c) $F_{\text {ENO }}$
d) None of the above

4. Which of the following statements is not true?
a) Side-effects of anti-TNF- $\alpha$ therapy can mimic a respiratory manifestation of IBD.
b) Eosinophilic infiltrates in bronchial biopsies are characteristic of IBD.
c) Non-caseating granulomatous inflammation can be found in lung biopsies of children with IBD.
d) Screening for tuberculosis is necessary before initiating anti-TNF- $\alpha$ therapy in children.

\section{Key points}

- Pulmonary involvement in paediatric IBD is rare, but may be missed. The spectrum of symptoms is broad and mimics other diseases. The differentiation between IBD-related and drug-induced respiratory manifestation can be challenging.

- The most important differential diagnoses of respiratory manifestations of IBD are infections and therapy-related side-effects.

- Lung function tests to monitor IBD under immunomodulatory therapy are not recommended.

- There is no gold standard to diagnose respiratory manifestations in children with IBD. Diagnostic tests should be chosen according to history and clinical presentation.

- Treatment of respiratory manifestations of IBD includes inhaled or oral corticosteroids and initiation or step-up of immunomodulatory IBD therapies. 


\section{Affiliations}

\section{Anja Jochmann, Daniel Trachsel, Jürg Hammer}

Division of Respiratory and Critical Care Medicine, University Children's Hospital Basel (UKBB), University of Basel, Basel, Switzerland.

\section{Conflict of interest}

A. Jochmann has nothing to disclose. D. Trachsel has nothing to disclose. J. Hammer has nothing to disclose.

\section{Suggested answers}

1. a.

2. c.

3. d.

4. b.

\section{References}

1. Collaborators GBDIBD. The global, regional, and national burden of inflammatory bowel disease in 195 countries and territories, 1990-2017: a systematic analysis for the Globa Burden of Disease Study 2017. Lancet Gastroenterol Hepatol 2020; 5: 17-30.

2. Braegger CP, Ballabeni P, Rogler D, et al. Epidemiology of inflammatory bowel disease: is there a shift towards onset at a younger age? J Pediatr Gastroenterol Nutr 2011; 53 : 141-144.

3. Dubinsky M. Special issues in pediatric inflammatory bowel disease. World J Gastroenterol 2008; 14: 413-420.

4. Kugathasan S, Judd RH, Hoffmann RG, et al. Epidemiologic and clinical characteristics of children with newly diagnosed inflammatory bowel disease in Wisconsin: a statewide population-based study. I Pediatr 2003; 143: 525-531.

5. Hildebrand H, Finkel Y, Grahnquist L, et al. Changing pattern of paediatric inflammatory bowel disease in northern Stockholm 1990-2001. Gut 2003; 52: 1432-1434.

6. Rabizadeh S, Dubinsky M. Update in pediatric inflammatory bowel disease. Rheum Dis Clin North Am 2013; 39: 789-799.

7. Lakatos L, Pandur T, David G, et al. Association of extraintestinal manifestations of inflammatory bowel disease in a province of western Hungary with disease phenotype: results of a 25-year follow-up study. World J Gastroenterol 2003; 9: 2300-2307.

8. Ji XQ, Wang LX, Lu DG. Pulmonary manifestations of inflammatory bowel disease. World J Gastroenterol 2014 20: 13501-13511

9. Black H, Mendoza M, Murin S. Thoracic manifestations of inflammatory bowel disease. Chest 2007; 131: 524-532.

10. Camus P, Colby TV. The lung in inflammatory bowel disease. Eur RespirJ 2000; 15: 5-10.

11. Betancourt SL, Palacio D, Jimenez CA, et al. Thoracic manifestations of inflammatory bowel disease. AJR Am J Roentgenol 2011; 197: W452-W456.

12. Vutcovici M, Brassard P, Bitton A. Inflammatory bowe disease and airway diseases. World J Gastroenterol 2016; 22: 7735-7741.

13. Vadlamudi NB, Navaneethan U, Thame KA, et al. Crohn's disease with pulmonary manifestations in children: 2 case reports and review of the literature. J Crohns Colitis 2013; 7 e85-e92.

14. Peradzynska J, Krenke K, Lange J, et al. Low prevalence of pulmonary involvement in children with inflammatory bowe disease. Respir Med 2012; 106: 1048-1054.

15. Barfield E, Deshmukh F, Slighton E, et al. Pulmonary manifestations in adolescents with inflammatory bowel disease. Clin Pediatr (Phila) 2020; 59: 573-579.

16. Higenbottam T, Cochrane GM, Clark TJ, et al. Bronchial disease in ulcerative colitis. Thorax 1980; 35: 581-585.

17. Mahadeva R, Walsh G, Flower CD, et al. Clinical and radiological characteristics of lung disease in inflammatory bowel disease. Eur Respir J 2000; 15: 41-48.

18. Ahmed KA, Thompson JW, Joyner RE, et al. Airway obstruction secondary to tracheobronchial involvement of asymptomatic undiagnosed Crohn's disease in a pediatric patient. Int J Pediat Otorhinolaryngol 2005; 69: 1003-1005.

19. Kuźniar T, Sleiman C, Brugiere O, et al. Severe tracheobronchia stenosis in a patient with Crohn's disease. Eur Respir J 2000 15: 209-212.
20. Henry MT, Davidson LA, Cooke NJ. Tracheobronchial involvement with Crohn's disease. EurJ Gastroenterol Hepatol 2001; 13: 1495-1497.

21. Russi A, Gurbani N, Rosen MJ, et al. Pediatric patient with ulcerative colitis-associated bronchiectasis. ACG Case Rep J 2020; 7: e00365.

22. Bentur L, Lachter J, Koren I, et al. Severe pulmonary disease in association with Crohn's disease in a 13-year-old girl. Pediatr Pulmonol 2000; 29: 151-154.

23. Krishnan S, Banquet $A$, Newman $L$, et al. Lung lesions in children with Crohn's disease presenting as nonresolving pneumonias and response to infliximab therapy. Pediatrics 2006; 117: 1440-1443.

24. Levenbrown Y, Tauber D, Hall OR, et al. Granulomatous lung disease as the initial presentation of Crohn disease. J Pediatr Gastroenterol Nutr 2009; 48: 487-490.

25. Kayser K, Probst F, Gabius HJ, et al. Are there characteristic alterations in lung tissue associated with Crohn's disease? Pathol Res Pract 1990; 186: 485-490.

26. Puntis JW, Tarlow MJ, Raafat F, et al. Crohn's disease of the lung. Arch Dis Child 1990; 65: 1270-1271.

27. Mahgoub LE, Puntis JW, Chetcuti PA, et al. Severe Crohn disease of the lung following colectomy. J Pediatr Gastroenterol Nutr 2007; 45: 477-479.

28. Calder CJ, Lacy D, Raafat F, et al. Crohn's disease with pulmonary involvement in a 3 year old boy. Gut 1993; 34: 1636-1638.

29. Al-Binali AM, Scott B, Al-Garni A, et al. Granulomatous pulmonary disease in a child: an unusual presentation of Crohn's disease. Pediatr Pulmonol 2003; 36: 76-80.

30. Maglione M, Aksamit T, Santamaria F. Paediatric and adult bronchiectasis: specific management with coexisting asthma COPD, rheumatological disease and inflammatory bowel disease. Respirology 2019; 24: 1063-1072.

31. Kirsner JB, Shorter RG. Recent developments in "nonspecific" inflammatory bowel disease (first of two parts). N Engl J Med 1982; 306: 775-785.

32. Aydin B, Songur Y, Songur N, et al. Investigation of pulmonary involvement in inflammatory bowel disease in an experimental model of colitis. Korean J Intern Med 2016; 31: 853-859.

33. Orchard TR, Chua CN, Ahmad T, et al. Uveitis and erythema nodosum in inflammatory bowel disease: clinical features and the role of HLA genes. Gastroenterology 2002; 123: 714-718.

34. Roussomoustakaki M, Satsangi J, Welsh K, et al. Genetic markers may predict disease behavior in patients with ulcerative colitis. Gastroenterology 1997; 112: 1845-1853.

35. Papanikolaou I, Kagouridis K, Papiris SA. Patterns of airway involvement in inflammatory bowel diseases. World Gastrointest Pathophysiol 2014; 5: 560-569.

36. Camus $P$, Piard F, Ashcroft T, et al. The lung in inflammatory bowel disease. Medicine (Baltimore) 1993; 72: 151-183.

37. Schleiermacher D, Hoffmann JC. Pulmonary abnormalities in inflammatory bowel disease. J Crohns Colitis 2007; 1: 61-69.

38. Corica D, Romano C. Biological therapy in pediatric inflammatory bowel disease: a systematic review. J Clin Gastroenterol 2017; 51: 100-110.

39. Imokawa S, Colby TV, Leslie KO, et al. Methotrexate pneumonitis: review of the literature and histopathological findings in nine patients. Eur Respir J 2000; 15: 373-381. 
40. Szymanska E, Dadalski M, Oracz G, et al. Safety profile of biologic therapy in Polish paediatric patients with Crohn's disease. Prz Gastroenterol 2015; 10: 164-168.

41. Keane J, Gershon S, Wise RP, et al. Tuberculosis associated with infliximab, a tumor necrosis factor alpha-neutralising agent. N EnglJ Med 2001; 345: 1098-1104.

42. Kaur N, Mahl TC. Pneumocystis jiroveci (carinii) pneumonia after infliximab therapy: a review of 84 cases. Dig Dis Sci 2007; 52 : 1481-1484.

43. Hirai F, Matsui T, Ishibashi Y, et al. Asymptomatic pulmonary cryptococcosis in a patient with Crohn's disease on infliximab: case report. Inflamm Bowel Dis 2011; 17: 1637-1638.

44. Toruner M, Loftus EV Jr, Harmsen WS, et al. Risk factors for opportunistic infections in patients with inflammatory bowel disease. Gastroenterology 2008; 134: 929-936.

45. Patel D, Madani S, Patel S, et al. Review of pulmonary adverse effects of infliximab therapy in Crohn's disease. Expert Opin Drug Saf 2016; 15: 769-775.

46. Spira A, Grossman R, Balter M. Large airway disease associated with inflammatory bowel disease. Chest 1998 ; 113: 1723-1726

47. Kelly MG, Frizelle FA, Thornley PT, et al. Inflammatory bowel disease and the lung: is there a link between surgery and bronchiectasis? Int J Colorectal Dis 2006; 21: 754-757.

48. Furlano RI, Basek P, Muller P, et al. Pulmonary function test abnormalities in pediatric inflammatory bowel disease. Respiration 2015; 90: 279-286.

49. Turner D, Levine A, Escher JC, et al. Management of pediatric ulcerative colitis: joint ECCO and ESPGHAN evidence-based consensus guidelines. J Pediatr Gastroenterol Nutr 2012; 55 : 340-361.

50. Ruemmele FM, Veres $G$, Kolho KL, et al. Consensus guidelines of ECCO/ESPGHAN on the medical management of pediatric Crohn's disease. J Crohns Colitis 2014; 8: 1179-1207.

51. Moeser $A$, Lerche $M$, Wirtz $H$, et al. Lungenbeteiligung bei chronisch-entzündlichen Darmerkrankungen [Aspects of pulmonary involvement in inflammatory bowel disease]. Internist (Berl) 2018; 59: 876-885.

52. Olpin JD, Sjoberg BP, Stilwill SE, et al. Beyond the bowel: extraintestinal manifestations of inflammatory bowel disease. Radiographics 2017; 37: 1135-1160.

53. Cozzi D, Moroni C, Addeo G, et al. Radiological patterns of lung involvement in inflammatory bowel disease. Gastroenterol Res Pract 2018; 2018: 5697846.

54. Krenke K, Peradzynska J, Lange J, et al. Inflammatory cytokines in exhaled breath condensate in children with inflammatory bowel diseases. Pediatr Pulmonol 2014; 49: 1190-1195

55. Huang Y, Lemberg DA, Day AS, et al. Markers of inflammation in the breath in paediatric inflammatory bowel disease. $J$ Pediatr Gastroenterol Nutr 2014; 59: 505-510.

56. El Amrousy DM, Hassan S, El-Ashry H, et al. Pulmonary function tests abnormalities in children with inflammatory bowel disease: is it common? J Pediatr Gastroenterol Nutr 2018; 67: 346-350.

57. Yammine S, Nyilas S, Casaulta C, et al. Function and ventilation of large and small airways in children and adolescents with inflammatory bowel disease. Inflamm Bowel Dis 2016; 22: 1915-1922.

58. Barbiellini Amidei C, Zingone F, Zanier L, et al. Risk of prevalent asthma among children affected by inflammatory bowel disease: a population-based birth cohort study. Int J Environ Res Public Health 2020; 17: 4255

59. Hayek AJ, Pfanner TP, White HD. Inflammatory bowel disease of the lung: the role of infliximab? Respir Med Case Rep 2015; 15: 85-88

60. Pedersen N, Duricova D, Munkholm P. Pulmonary Crohn's disease: a rare extra-intestinal manifestation treated with infliximab. J Crohns Colitis 2009; 3: 207-211.

61. Marvisi M, Fornasari G. Il polmone è un organo bersaglio nella malattia infiammatoria intestinale? [ls the lung a target organ in inflammatory bowel disease?] Recenti Prog Med 2001; 92: 774-777. 